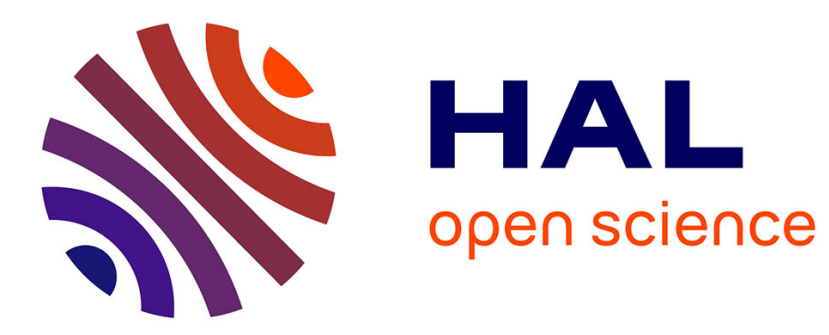

\title{
Testing of polymeric foams at high and medium strain rates
}

\author{
H. Zhao
}

\section{To cite this version:}

H. Zhao. Testing of polymeric foams at high and medium strain rates. Polymer Testing, 1997, 16, pp.507-516. 10.1016/S0142-9418(97)00012-3 . hal-00111587

\section{HAL Id: hal-00111587 \\ https://hal.science/hal-00111587}

Submitted on 4 Mar 2019

HAL is a multi-disciplinary open access archive for the deposit and dissemination of scientific research documents, whether they are published or not. The documents may come from teaching and research institutions in France or abroad, or from public or private research centers.
L'archive ouverte pluridisciplinaire HAL, est destinée au dépôt et à la diffusion de documents scientifiques de niveau recherche, publiés ou non, émanant des établissements d'enseignement et de recherche français ou étrangers, des laboratoires publics ou privés. 


\title{
Testing of Polymeric Foams at High and Medium Strain Rates
}

\author{
Han Zhao \\ Laboratoire de Mécanique des Solides, Ecole Polytechnique, 91128 Palaiseau, France
}

\begin{abstract}
Experimental data of the behaviour of polymeric foams at high and medium strain rates is required in the crash simulations developed in the automotive industry. This paper presents an investigation of particular difficulties in the testing of polymeric foams, using a Split Hopkinson Pressure Bar (SHPB). On the one hand, viscoelastic bars are used to improve the impedance ratio between the bar and the specimen and consequently the accuracy of measurements. A generalised two-gauge method is used to extend this measurement to the complete range of strain (up to $80 \%$ of nominal strain), and allows also for performing tests at medium strain rates $\left(5-50 s^{-1}\right)$. On the other hand, the assumption of the homogeneity of the strain and stress fields in the specimen is studied, using a high speed photographic system.
\end{abstract}

\section{INTRODUCTION.}

Mechanical properties of polymeric foams are often involved in the study of crash worthiness in the automotive industry. For example, anthropomorphic dummies have been developed to investigate the response of the human body and to analyse the interaction with the passenger compartment. Polymeric foams are recurrent both in the crash dummies and in the passenger compartment. Experimental car crash investigation offers currently appreciable capabilities but requires a car prototype. To evaluate the occupant protection in the early phases of car design by numerical crash simulations, modelling of 
the mechanical behaviour of polymeric foams and especially their rate sensitivity is indispensable. Accurate experimental data on polymeric foams is then needed.

The quasi-static behaviour of the polymeric foams can be obtained with the ordinary testing machine and some constitutive models are proposed. ${ }^{1}$ The measurement of the behaviour of polymeric foams at relatively high strain rates has been an interest of investigators since the 1960s. Lacey ${ }^{2}$ and Traegar $^{3}$ have reported their results at high and medium strain rates using falling weight techniques. As they have indicated, the measurement using those techniques is not accurate.

The Split Hopkinson Pressure Bar (SHPB) is a widely used experimental technique to study constitutive laws of materials at high strain rates. ${ }^{4,5}$ This technique, initially developed for the testing of metallic materials, has gained great popularity in the past decades and is also used for many non-metallic materials (rocks, concrete, polymer, etc) ${ }^{6}$ Rinde and Hoge ${ }^{7,8}$ have performed tests on polymeric forms at high strain rate with SHPB. However, because of a too weak resistance of foams, the specimen stress is measured by quartz crystals which are not sufficiently accurate at the early stage of loading so that the apparent Young's modulus and yield stress have not been measured. Furthermore, the limitation of measuring duration of the classical SHPB makes the maximum measured strain during the test less than $10 \%$.

In this paper, particular difficulties of the testing of polymeric foams at high and medium strain rates are investigated. The use of viscoelastic SHPB bars permits an accurate measurement at high strain rates and the generalised two-gauge method extends this measurement to a large strain range, and this allows an accurate measurement at medium strain rates $\left(5-50 \mathrm{~s}^{-1}\right)$. A high speed photographic device is developed to analyse the validity of the assumption of the homogeneity, of strain and stress fields in the specimen, which is incorporated in the classical SHPB analysis.

\section{MEASURING PROBLEMS IN TESTING ON FOAMS}

\subsection{Impedance ratio and the use of a viscoelastic SHPB setup}

A typical SHPB setup is composed of the long input and output bars with a short specimen placed between them (Fig. 1). The impact of the projectile at the free end of the input bar develops a compressive longitudinal incident wave $\epsilon_{i}(t)$. Once it arrives at the bar specimen interface, a reflected wave $\epsilon_{r}(t)$ is developed in the input bar, whereas a transmitted wave $\epsilon_{t}(t)$ is developed in the output bar. From those basic experimental data (incident, reflected and 


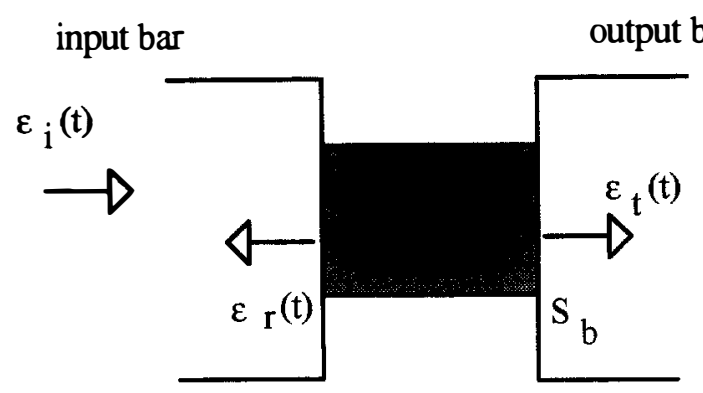

Fig. 1. Scheme of SHPB set-up.

transmitted waves), forces and velocities at both faces of the specimen can be calculated.

$$
\begin{array}{cc}
F_{\text {input }}(t)=S_{b} E_{b}\left(\epsilon_{i}(t)+\epsilon_{r}(t)\right) & V_{\text {input }}(t)=C_{b}\left(\epsilon_{i}(t)-\epsilon_{r}(t)\right) \\
F_{\text {output }}(t)=S_{b} E_{b} \epsilon_{t}(t) & \text { and }
\end{array}
$$

where $E_{b}, S_{b}, C_{b}$ denote Young's modulus, the cross-sectional area and wave speed of the bar.

Using the classical assumption of homogeneous stress and strain fields in the specimen, an average stress-strain relation is then given as follows. ${ }^{5}$

$$
\begin{gathered}
\dot{\epsilon}_{s}(t)=\frac{2 C_{b}}{l_{s}} \epsilon_{r}(t) \\
\sigma_{s}(t)=-\frac{E_{b} S_{b}}{S_{s}} \epsilon_{t}(t)
\end{gathered}
$$

where $l_{\mathrm{s}}$ and $S_{\mathrm{s}}$ are the length and the cross-sectional area of the specimen.

Nevertheless, one must overcome a major difficulty, which consists of a too low impedance ratio between the foam specimens and metallic split bars. Indeed, the partition of the reflected wave and that of the transmitted wave depend on the actual impedance ratio $r$ between specimen and bars ( $r$ not being constant during the test).

$$
\begin{aligned}
\epsilon_{r}(t) & =-\frac{1-r}{1+r} \epsilon_{i}(t), \epsilon_{t}(t)=\frac{r}{(1+r)^{2}} \epsilon_{i}(t) \\
\text { with } r & =S_{s} \rho_{s} C_{s} / S_{b} \rho_{b} C_{b}
\end{aligned}
$$

where $S_{b}, \rho_{b}, C_{b}$ and $S_{s}, \rho_{s}, C_{s}$, are the cross-section, the density and the wave speeds of the bar and the specimen, respectively. 
As the impedance of foams is very small (about $0.1 \%$ of the impedance of the bars), the transmitted wave $\epsilon_{t}(t)$ is weak, and $\epsilon_{r}(t)$ is almost equal to $-\epsilon_{i}(t)$. This then leads to imprecise measurements of the input force, output forces and output velocity [eqn (1)]. Consequently, in earlier tests on foams using ordinary metallic bars, an additive measuring device such as quartz crystals is used. ${ }^{7,8}$

The use of low impedance bars which are generally viscoelastic is then proposed. Two $3 \mathrm{~m}$ long nylon bars of $40 \mathrm{~mm}$ diameter (density being $1200 \mathrm{~kg} / \mathrm{m}^{3}$ and wave speed $1700 \mathrm{~m} / \mathrm{s}$ ) are used in our laboratory; it provides an improvement of about 20 times the impedance ratio, in comparison with a classical steel bar (density being $7850 \mathrm{~kg} / \mathrm{m}^{3}$ and wave speed 5000).

The use of viscoelastic bars in a SHPB setup introduces complications such as the wave dispersion in a viscoelastic bar, the calculation of stress and particle velocity from the measured strain and the projectile length limitations. ${ }^{9}$ The correction of dispersion effects based on the analysis of wave propagation in an infinite viscoelastic cylindrical bar is used. ${ }^{10,11}$ With precautions in the data processing, the nylon bar provides a satisfactory measuring precision. ${ }^{12}$

A typical nylon bar test on foams (diameter and length of the specimen being $40 \times 40 \mathrm{~mm}$, respectively) is shown in Fig. 2. The stress and strain rate curves are obtained using the classical SHPB analysis (eqn (2), its validity will be discussed in Section 3). It is illustrated that the strain rate during the test is quite constant and the apparent elastic modulus and yield stress are

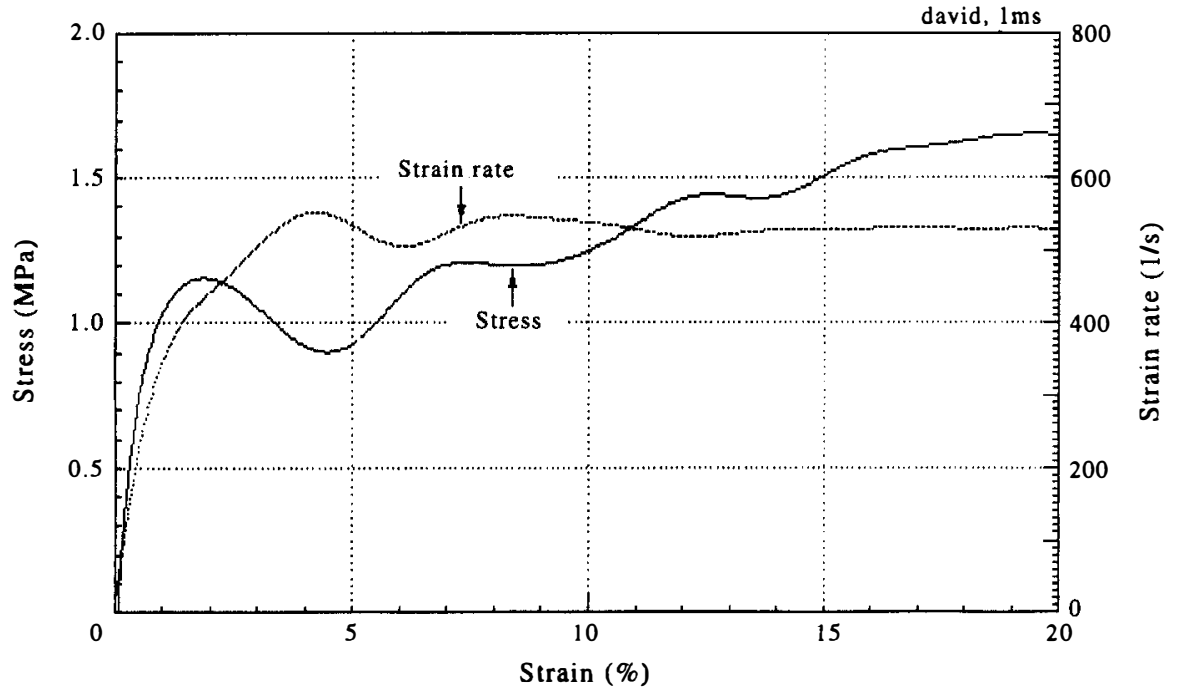

Fig. 2. Typical tests on polymeric foams. 
obtained. Nevertheless, the measurable maximum strain is about $20 \%$ at a strain rate of $500 \mathrm{~s}^{-1}$.

\subsection{Limitation of measuring duration and the use of the generalised two-gauge method}

One particular feature of foam testing is the need to achieve a large maximum strain (up to 80\%) in order to study the densification part of the response, associated with a significant increase of the stress. It is shown above that the SHPB test cannot provide such strains, even if such strains are easily reached after the measuring phase, because of the very low resistance of foams.

This is because the measuring duration of SHPB is limited. Indeed, the measuring technique using bars is based on the following principle. According to the elastic wave propagation theory, the stress, the strain and the particle velocity associated with a single wave are proportional to each other; and the superposition principle implies that the stress, the strain and the particle velocity at any cross-section can be considered as the algebraic sum of those values associated with the two elementary waves propagating in opposite directions at this cross-section. Such a technique requires then a separate measurement of each single wave propagating in the bar, but the measured strain is due to the contribution of two elementary waves.

However, an observing window could exist when the reflection has not come back and superimposed with the incident impulse. The SHPB uses this observing window so that its observation duration $\Delta T$ depends on the length of the bar and is limited to $\Delta T \leq L C, C$ being the wave speed and $L$ the length of the bar. Consequently, the measurable strain is limited for a given average strain rate $\left(\epsilon_{\max }<\dot{\epsilon} \Delta T\right)$.

To overcome this measuring limitation, one has to investigate the multiple reflections in bars. ${ }^{13} \mathrm{~A}$ two-gauge method has been reported to separate the two waves in elastic bars, ${ }^{14}$ and it can be extended to separate the two elementary waves in the case of a viscoelastic bar where the correction of wave dispersion is indispensable. ${ }^{15}$

In practice, the strain history in each bar is recorded by two strain gages at distinct points A and B. Since the recorded strain is the sum of the contributions of the two waves propagating in opposite directions, we have:

$$
\begin{aligned}
& \epsilon_{A}(t)=\epsilon_{a s c A}(t)+\epsilon_{\text {desA }}(t) \\
& \epsilon_{B}(t)=\epsilon_{a s c B}(t)+\epsilon_{\text {des } B}(t)
\end{aligned}
$$

where $\epsilon_{\text {ascA }}(t), \epsilon_{\text {asc } B}(t), \epsilon_{\text {desA }}(t), \epsilon_{\text {desB }}(t)$ are waves propagating in the 'ascending' direction (artificially defined) and those in the 'descending' direction at $\mathrm{A}$ and $\mathrm{B}$. 
We break down the two strain measurements $\epsilon_{A}(t)$ and $\epsilon_{B}(t)$ into time intervals of $\Delta t$ corresponding to the time for a round-trip between $\mathrm{A}$ and $\mathrm{B}$ (Fig. 3 ). The first interval at point A contains the single 'ascending' wave induced at one end of the bar before it reflects at the other end. Knowing how the waves propagate, we shift the interval to point B and focus on the 'ascending' wave at that point. Using eqn (4) we can now calculate the 'descending' wave at point $\mathrm{B}$ for the same interval. Continuing in this way, both the 'ascending' and the 'descending' waves can be calculated for all the intervals. The stresses and the particle velocities at each section of bar can be then calculated from the two virtually separated waves. In this way, an unlimited duration of measurement can be obtained. It leads to the measurement in the complete range of strain for testing on foams.

The use of such an unlimited measuring technique can be also applied to perform tests at medium strain rates. In studies related to passive security in the automotive industry, strain rates involved in a crash situation are approximately situated between $5 \mathrm{~s}^{-1}$ and $1000 \mathrm{~s}^{-1}$, and mostly in the lower part. It is important to have experimental data of foams at medium strain rates (5$50 \mathrm{~s}^{-1}$ ) which are generally performed with a fast hydraulic machine where a suitable measuring technique, often very expensive, is needed to obtain reliable signals. ${ }^{16}$

Figure 4 shows the rate sensitivity of foams in its complete range of strain. The test at $250 \mathrm{~s}^{-1}$ is performed with a nylon SHPB. The generalised twogage method provides four loading-unloading cycles corresponding to the round-trip of input impulse in the input bar, whereas the classical analysis

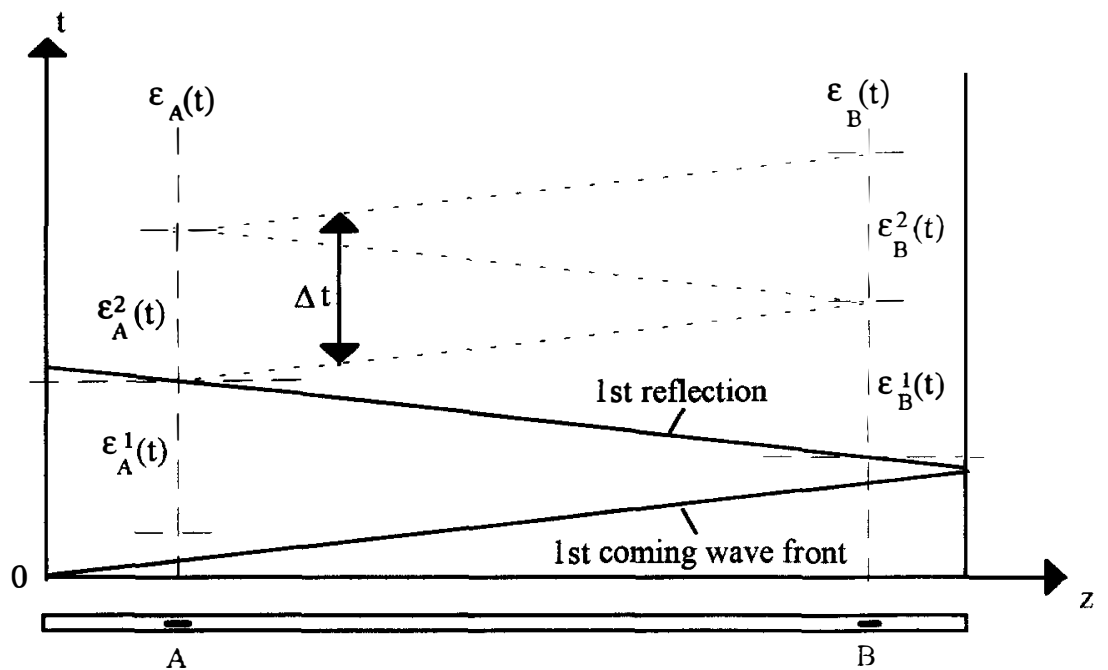

Fig. 3. Scheme of the separation of waves. 


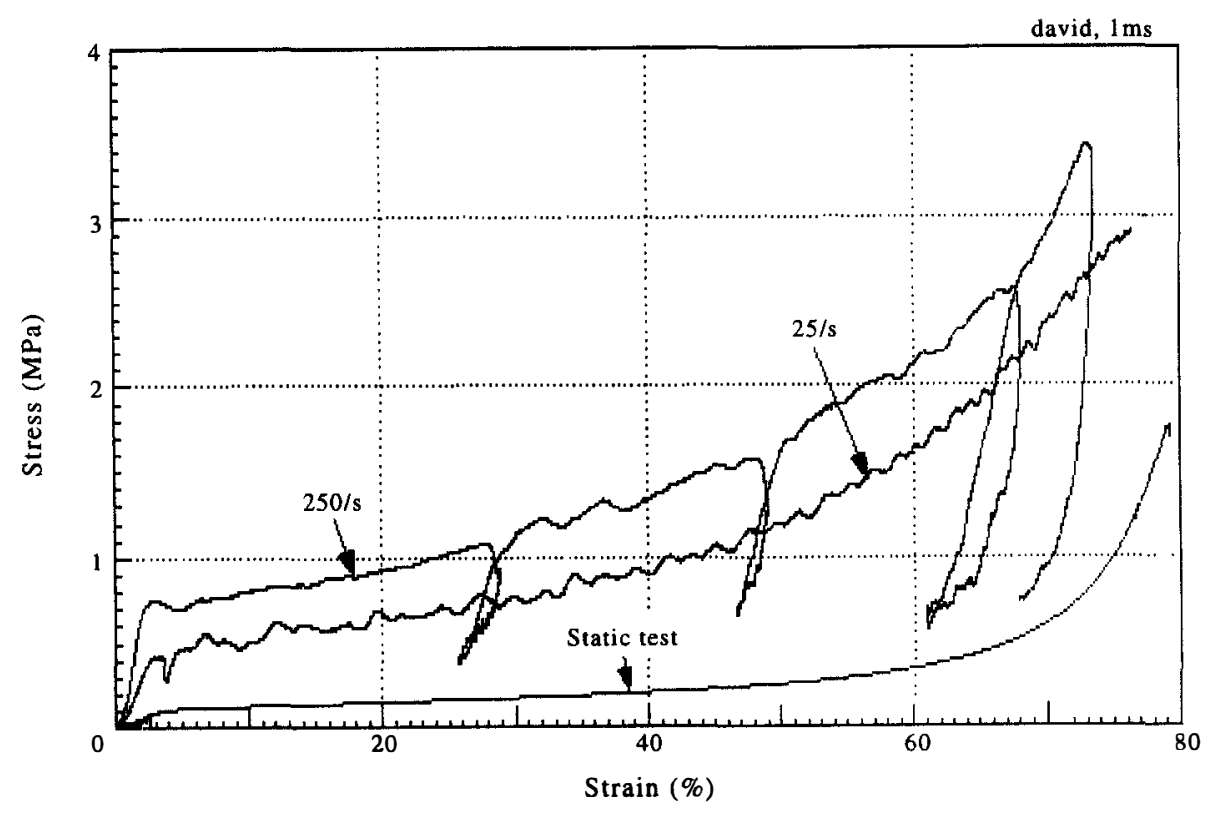

Fig. 4. Rate sensitivity of foams in the complete range of strain.

gives only half of a cycle. The test at $25 \mathrm{~s}^{-1}$ is performed by a so-called 'slowbar' technique which uses the bars as a measuring device and a high speed oil jack as a loading device. ${ }^{17}$

\section{DISCUSSION ON THE CLASSICAL ANALYSIS OF SHPB TEST ON FOAMS}

The stress-strain relation presented above is on the basis of the classical SHPB analysis which is correct only when the homogeneity of stress and strain fields is verified. ${ }^{5}$ In order to study if there is really the uniformity of strain fields in the specimen, a high speed image acquisition device was developed to observe qualitatively the specimen during tests. This setup gives four images at the chosen time delay with an exposition time up to 1 image per microsecond (Fig. 5). It is shown that there is not always axial uniformity of strain field in the specimen (Fig. 6), at least at the early stage of the loading. It is rather easy to prove that the stress field in the specimen is not uniform either. The use of viscoelastic nylon bars provides an accurate measurement of input force, and Fig. 7 shows that the input force is about twice the output one at the early stage of the test.

In this paper, it is proposed to use the measurement of the input force as a tool to check if the assumption homogeneity can be used. On the one hand, 


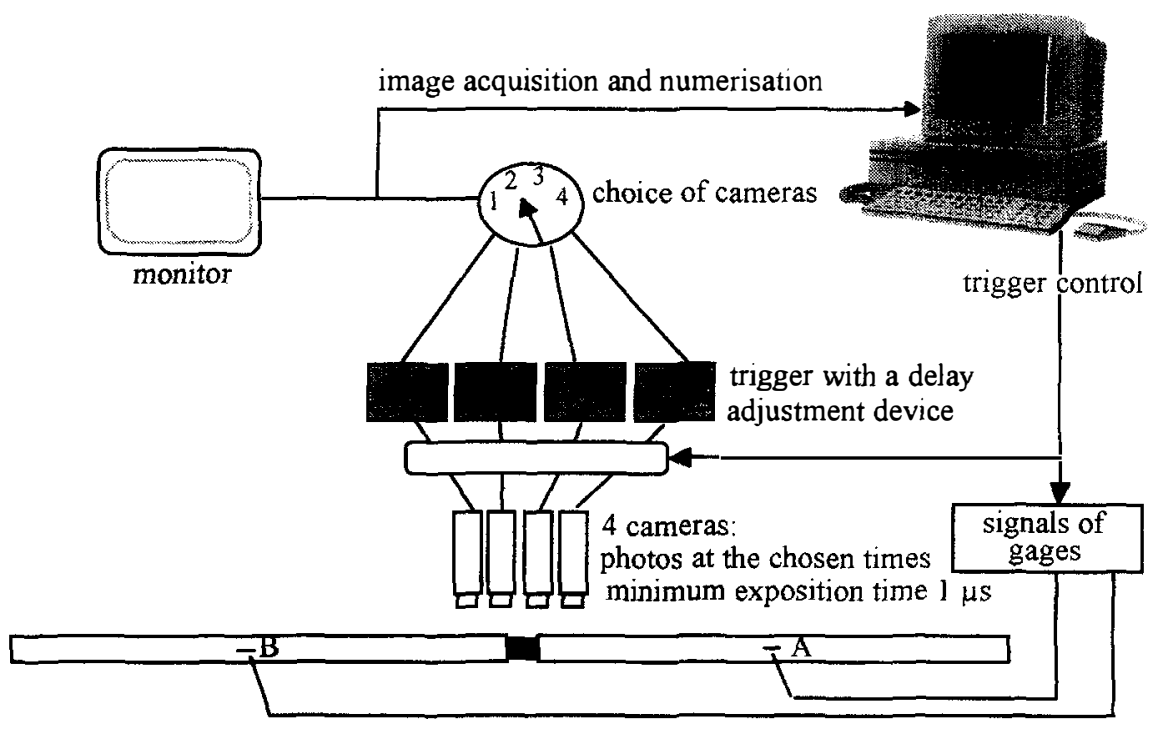

Fig. 5. Scheme of image acquisition device.
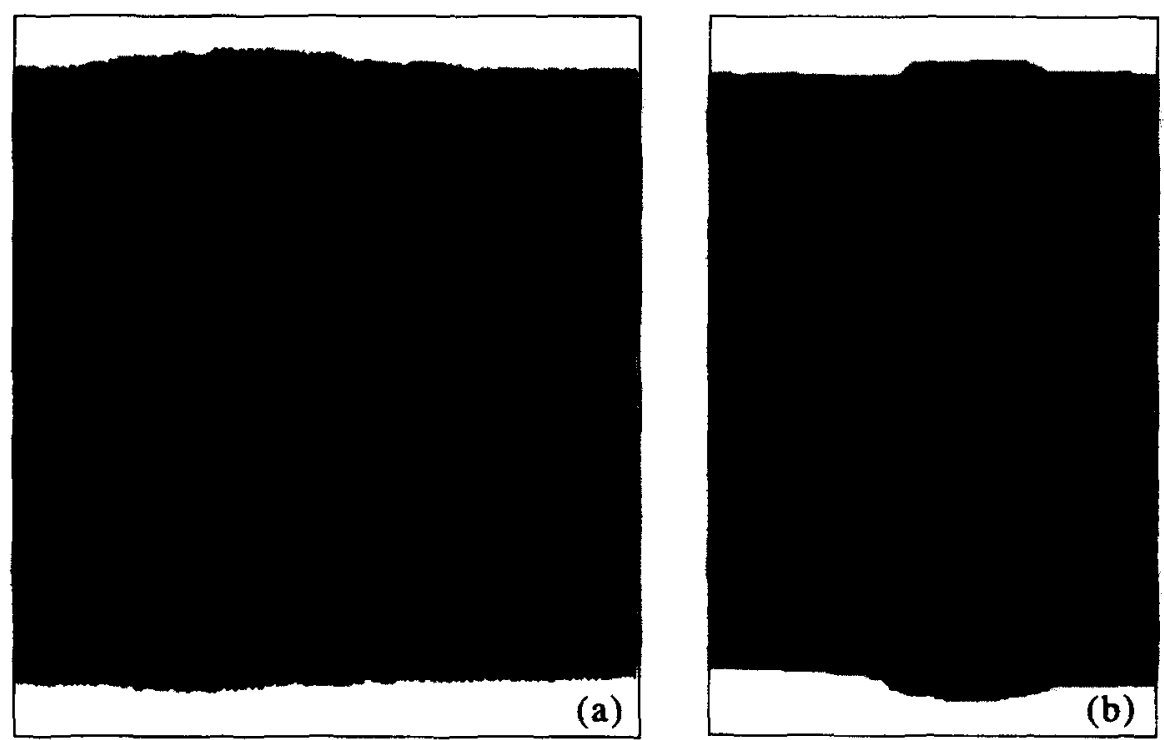

Fig. 6. Image of the strain field in a foam specimen: (a) at the early stage of loading; (b) at the end of loading. 


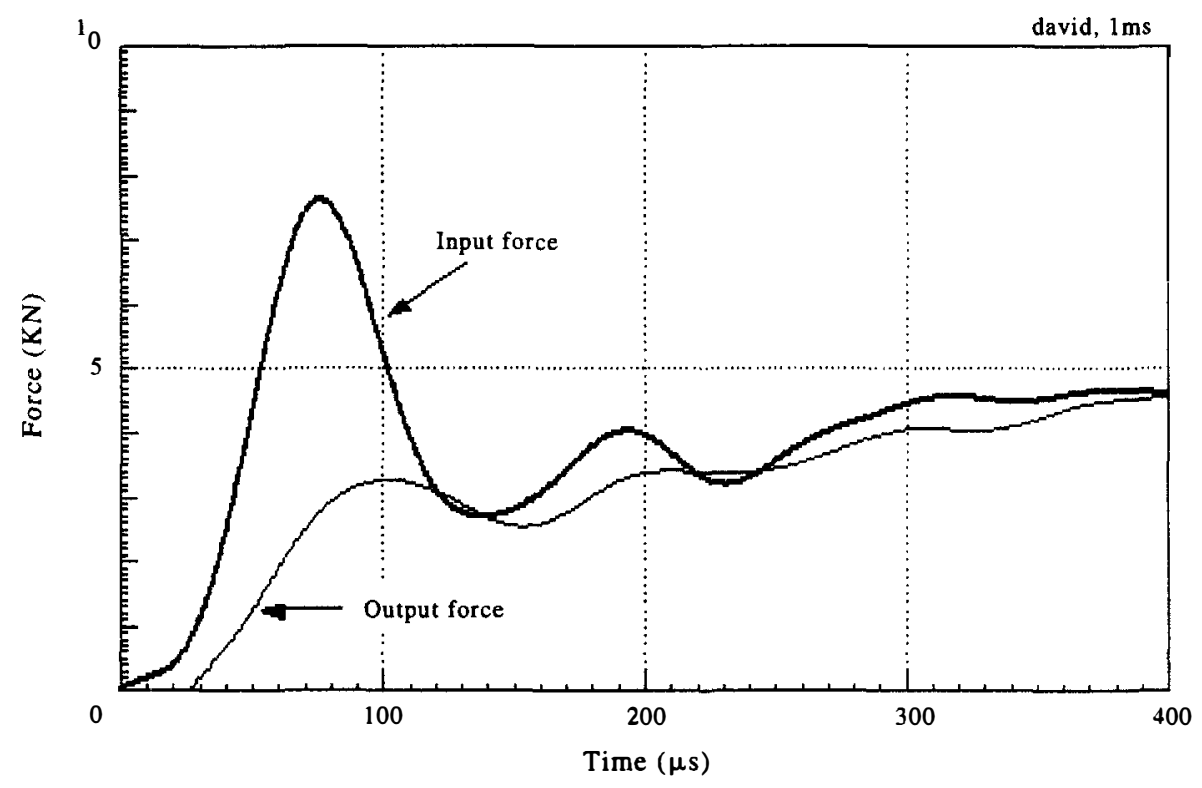

Fig. 7. Input and output forces for a foam specimen.

when the difference between the two forces is small, the conventional SHPB formulae can be used. On the other hand, when this difference is quite significant, the measurement of the forces and particle velocities at two bar-specimen interfaces can be used to produce the information on material behaviour in another way. Indeed, a SHPB test provides superabundant measurements that are forces and velocities at both ends of the specimen. In order to obtain the material behaviour, an identification technique based on an inverse calculation method can be used in such a case, as used in the case of concrete and rocks. ${ }^{6}$ It is assumed that an appropriate form of the material behaviour is known, with parameters to be determined. Using a part of data as input data, (velocities for example), another part of data (the two forces) is used to determine the parameters that give the best fit between the measured and calculated forces. Theoretical analyses ${ }^{18}$ show that this inverse technique can provide good results if an appropriate form of the material behaviour of foams is found.

\section{CONCLUSIONS}

In this paper, the measuring imprecision due to the weak impedance ratio, the limitation of maximum strain, and the validity of assumption of homogeneity of stress and strain fields are the main problems in the testing on polymeric 
foams using SHPB. Accurate experimental data at high and medium strain rates is obtained in their complete range of strain (up to 80\%), using a viscoelastic SHPB setup and a generalised two-gage method. The inverse method can be applied whenever there is no equilibrium of forces in the specimen.

\section{REFERENCES}

1. Gibson, L. J. and Ashby, M. R., Cellular Solids. Pergamon Press, 1988.

2. Lacey, R. M., Fifth Int. Symp. on High Speed Testing, Boston, 1965, pp. 99-105.

3. Traegar, R. K., J. Cellular Plastics, 1967, 3, 405-418.

4. Hopkinson, B., Phil. Trans. Roy. Soc., 1914, A213, 437-452.

5. Kolsky, H., Proc. Phys. Soc. London, 1949, B62, 676-700.

6. Zhao, H. and Gary, G., Int. J. Solid. \& Structures, 1996, 33, 3363-3375.

7. Rinde, J. A. and Hoge, K. G., J. Appl. Polym. Sci., 1971, 15, 1377-1395.

8. Rinde, J. A. and Hoge, K. G., J. Appl. Polym. Sci., 1972, 16, 1409-1415.

9. Zhao, H., PhD thesis, ENPC, Paris, 1992.

10. Davies, R. M., Phil. Trans. Roy. Soc., 1948, A240, 375-457.

11. Zhao, H. and Gary, G., J. Mech. Phys. Solids, 1995, 43, 1335-1348.

12. Zhao, H., Gary, G. and Klépaczko, J. R., Int. J. Impact Engng, 1997, in press.

13. Campbell, J. D. and Duby, J., Proc. R. Soc. Lond., 1956, A236, 24-40.

14. Lundberg, B. and Henchoz, A., Exper. Mech., 1977, 17, 213-218.

15. Zhao, H. and Gary, G., C. R. Acad. Sci. Paris, 1994, 319(II), 987-992.

16. Holzer, A. J., Int. J. Mech. Sci., 1978, 20, 553-560.

17. Zhao, H. and Gary, G., J. Mech. Phys. Solids, 1997, in press.

18. Rota, L., Proc. 2nd Int. Symp. On Inverse Problems - ISIP'94, ed. H. B. Bui, M. Tanaka et al. A. A. Balkema, Rotterdam, 1994, pp. 157-164. 